\title{
Sex Differences in the Temporalis Tendon- Aponeurotic Complex: An in vivo MRI Macroscopic Analysis in Children and Adolescents
}

\author{
Rachelle Isidro ${ }^{a}$ lacopo Cioffia, b \\ aCentre for Multimodal Sensorimotor and Pain Research, Faculty of Dentistry, University of Toronto, \\ Toronto, ON, Canada; ${ }^{b}$ Department of Dentistry, Mount Sinai Hospital, Toronto, ON, Canada
}

\section{Keywords}

Temporal muscle · Magnetic resonance imaging $\cdot$ Tendon · Aponeurosis

\begin{abstract}
The tendon-aponeurosis complex (TAC) of the temporalis dissipates forces produced during function. Abnormally reduced temporalis TACs have been found in individuals with chronic muscular temporomandibular disorders - a painful musculoskeletal condition that is more frequent in women than men. Whether there are sex differences in the temporalis TAC in healthy individuals is currently unknown. Here, we characterized and measured the temporalis TAC in healthy male and female young individuals between 5 and 15 years old to determine whether the volume of the temporalis TAC and the TAC-to-muscle ratio are different between males and females. We collected MRI studies from 90 healthy individuals, including equal numbers (15 $\mathrm{M}$ and $15 \mathrm{~F}$ ) of young children (ages 5-7), children (9-11), and adolescents (13-15) and segmented the right temporalis and its TAC using software. Using general linear models, we tested the effect of sex, age, and their interaction on the volumes of the temporalis and its TAC, and the TAC-to-muscle ratio. The vol-
\end{abstract}

umes of the temporalis and its TAC increased with age (both $p<0.001)$ and were not affected by sex $(p=0.252$ and $p=$ $0.179)$ or by the interaction sex-by-age $(p=0.079$ and $p=$ 0.095 , respectively). The TAC-to-muscle ratio did not change significantly with age ( $p=0.655)$ and was not affected by sex $(0.438)$ or by the interaction sex-by-age $(0.524)$. We provide, for the first time, volumetric data of the temporalis TAC in children and adolescents. The volumes of the temporalis TAC and the TAC-to-muscle ratio are not different between male and female individuals until the age of 15 .

(c) 2021 The Author(s)

Published by S. Karger AG, Basel

\section{Introduction}

The human temporalis is a broad and fan-shaped muscle situated within the temporal fossa. It plays a major role in all jaw functional activities such as chewing, biting, drinking, swallowing and speaking [Farella et al., 2008], and is also active during nonfunctional oral activities, such as tooth clenching and grinding [Farella et al., 2008], which are risk factors for painful muscular temporomandibular disorders (mTMD) [Huang et al., 2002; Michelotti et al., 2010; Benoliel et al., 2011; Slade et al., 2016].
(C) 2021 The Author(s)

Published by S. Karger AG, Basel

This is an Open Access article licensed under the Creative Commons Attribution-NonCommercial-4.0 International License (CC BY-NC) (http://www.karger.com/Services/OpenAccessLicense), applicable to the online version of the article only. Usage and distribution for commercial purposes requires written permission.
Correspondence to:

Iacopo Cioffi, iacopo.cioffi@ dentistry.utoronto.ca 
The muscle fibers of the temporalis converge in a thick tendon inserting at the coronoid process, the anterior border of the mandibular ramus, and the retromolar pad on the mandibular body [Geers et al., 2005]. The temporalis tendon and its fan-shaped aponeurosis form a tendon-aponeurosis complex (TAC), which divides the muscle into a superficial and deep temporalis [Geers et al., 2005; Lee et al., 2012]. Cadaveric studies have identified at least 3 functional neuromuscular compartments in the superior part of the temporalis and 2 functional neuromuscular partitions in the anterior and posterior regions of the inferior part of the temporalis [Chang et al., 2013], which suggests that different portions of the temporalis may be activated selectively during function [Blanksma and van Eijden, 1995; Blanksma et al., 1997].

The temporalis TAC protects the muscle from contraction-induced or high-strain injuries by storing and dissipating strain energy [Sharma and Maffulli, 2006]. Using finite element models, Fiorentino and Blemker [2014] showed that the relative dimensions of muscle and aponeurosis influence internal muscle tissue strains: peak local tissue strain increased as the proximal aponeurosis width of the biceps femoris long head narrowed and the muscle width widened. Therefore, a smaller TAC may predispose to musculoskeletal injuries and contribute to musculoskeletal pain. Of note, a reduced temporalis TAC contributing to a decreased TAC-to-muscle volume ratio (TAC:muscleR) was found in individuals with chronic facial musculoskeletal pain due to mTMD [Moayedi et al., 2020].

As mTMD is more prevalent in female than male individuals [Fillingim and Maixner, 2000; Slade et al., 2016], it could be hypothesized that females may have smaller temporalis TAC than males contributing to decreased TAC:muscleR.

To date, the temporalis TAC has been minimally studied in vivo [Moayedi et al., 2020], and possible sex-related anatomical differences have not been investigated. In this study, we aimed to determine whether the volume of the temporalis TAC is different between healthy male and female young individuals between 5 and 15 years old. We hypothesized that females would have a smaller temporalis TAC compared to male individuals, contributing to a smaller TAC:muscleR.

\section{Materials and Methods}

\section{Study Sample}

MRI head scans of healthy individuals aged between 5 and 15 years old were obtained from the CMI-HBN data repository (http:// fcon_1000.projects.nitrc.org/indi/cmi_healthy_brain_network/).
CMI-HBN collects imaging and clinical data of young volunteers and share this de-identified data with the global scientific community to study brain development and promote discovery of biomarkers of mental health disorders in the developing brain. This database provided the subjects' age and sex, psychiatric, behavioral, cognitive information, and medical history, along with a structural T1-weighted image of the neurocranium, which includes the temporalis of the left and right sides.

Excluded subjects for this study were those with motor disorders, neurodevelopmental or neurobehavioral disorders, syndromes, craniofacial anomalies or orofacial pain conditions. The evaluation of the exclusion criteria was based on the information provided by the CMI-HBN repository for each MRI study.

We collected MRI studies from a convenience sample of 90 individuals, divided in 3 age groups, each including 15 males and 15 females, as following: young children: ages 5-7 (mean age \pm SD: 6.1 \pm 0.5 years); children: ages $9-11$ (mean age \pm SD: $9.8 \pm 0.6$ years), and adolescents: ages $13-15$ (mean age \pm SD: $14.1 \pm 0.6$ years). The MRI studies were randomly selected within each age range. The age range of the adolescents was named " $13-15$ " here for practicality, although the oldest subjects ( $>14.9$ years old) in this group did not reach the 15.0 years old limit.

\section{MRI Assessment}

As per CMI-HBN reporting, data were acquired using a $1.5 \mathrm{~T}$ MRI system (Siemens, Avanto, Germany). High-resolution, threedimensional (3D), T1-weighted anatomical image volumes were acquired, covering the entire brain and included both the right and left temporalis. The scanning parameters were as follows: echo time: $2.4 \mathrm{~ms}$; repetition time: 5,200 ms; field of view $220 \times 220 \mathrm{~mm}$; voxel dimensions: $1.72 \times 1.72 \times 1.8 \mathrm{~mm}$.

\section{Regions of Interest Definition}

Based on a previous study from our research lab which included both MR assessments and an anatomic verification on a cadaver [Moayedi et al., 2020], the superior boundary of the TAC region of interest (ROI) in MRI images was set at the level of the superior orbital rim on the right side. The inferior boundary was set to the first axial slice where the higher signal cancellous bone within the right coronoid process of the mandible was visualized (Fig. 1). The anterior, posterior, medial, and lateral boundaries of the TAC ROI were the anterior, posterior, medial, and lateral surfaces of the right temporalis, respectively.

The temporalis ROI included the entire right temporalis and was limited inferiorly at the first axial MRI slice where the higher signal cancellous bone within the coronoid process of the mandible was visualized.

\section{Quantification of Temporal Muscle and Temporalis TAC}

User-guided segmentation of the right temporalis and its TAC were carried out in ITK-SNAP (www.itksnap.org) [Yushkevich et al., 2006] with a Huion WH1409 (Shenzhen, China) graphic tablet (Fig. 1). All images were segmented by a single examiner (R.I.) who was blinded to participants' age and sex (dataset masking). Both the temporal muscle and its TAC were segmented using a semiautomatic segmentation pipeline. Therefore, ROIs of the structures were created in a semi-automatic way, and manually refined. Each MR study was verified in all orthogonal planes. Any uncertainty in temporal muscle/TAC segmentation was discussed with a second examiner (I.C.), with expertise in masticatory muscle MRI anato- 
Fig. 1. Segmentation of the right temporalis (orange) and its tendon-aponeurosis complex (TAC, purple), and 3D rendering. A: anterior. P: posterior. R: right. L: left. The horizontal red lines indicate the upper and lower boundaries of the TAC region of interest in MR image.

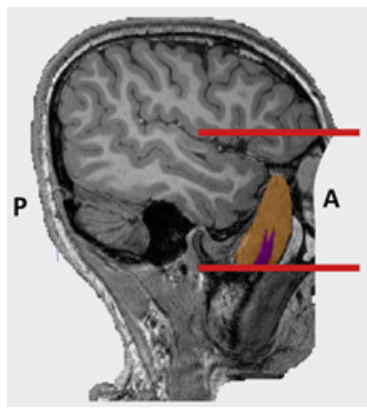

Table 1. Descriptive statistics

\begin{tabular}{llccc}
\hline & & Total sample & Males & Females \\
\hline Young children & Temporalis volume $\left(\mathrm{mm}^{3}\right)$ & $20,356.44 \pm 1,365.54$ & $20,531.36 \pm 1,274.64$ & $20,181.52 \pm 1,473.88$ \\
$N=30$ (age 5-7) & TAC volume $\left(\mathrm{mm}^{3}\right)$ & $1,303.21 \pm 229.08$ & $1,261.01 \pm 215.95$ & $1,345.40 \pm 241.37$ \\
& TAC:muscleR $(\%)$ & $6.01 \pm 0.95$ & $5.77 \pm 0.82$ & $6.25 \pm 1.04$ \\
\hline Children & Temporalis volume $\left(\mathrm{mm}^{3}\right)$ & $24,934.20 \pm 3,731.56$ & $25,170.12 \pm 3,288.29$ & $24,698.28 \pm 4,232.20$ \\
$N=30$ (age 9-11) & TAC volume $\left(\mathrm{mm}^{3}\right)$ & $1,583.10 \pm 328.12$ & $1,624.05 \pm 292.99$ & $1,542.16 \pm 365.48$ \\
& TAC:muscleR $(\%)$ & $5.98 \pm 0.97$ & $6.07 \pm 0.87$ & $5.89 \pm 1.07$ \\
\hline Adolescents & Temporalis volume $\left(\mathrm{mm}^{3}\right)$ & $36,418.80 \pm 6,714.89$ & $38,782.87 \pm 5,948.71$ & $34,054.72 \pm 6,785.06$ \\
$N=30$ (age 13-15) & TAC volume $\left(\mathrm{mm}^{3}\right)$ & $2,369.05 \pm 447.15$ & $2,514.67 \pm 453.42$ & $2,223.42 \pm 403.90$ \\
& TAC:muscleR $(\%)$ & $6.16 \pm 0.91$ & $6.15 \pm 1.1$ & $6.18 \pm 0.71$ \\
\hline
\end{tabular}

TAC, tendon-aponeurosis complex; TAC:muscleR, TAC-to-muscle ratio. Values are presented as means \pm SD.

my. The volumetric data were computed automatically by the software by using voxel volume and number of selected voxels for each ROI. Volumetric data for each ROI were then recorded for analysis.

\section{Intra-Rater Reliability}

To test the reproducibility of the segmentation method, the ROIs of 12 randomly selected MRI scans were segmented a second time - 1 month after the initial segmentation by the same examiner (R.I.). Intraclass correlation coefficients (ICCs) were calculated to measure intra-rater reliability.

\section{Statistical Analysis}

The primary outcome measures of each MRI segmentation were the TAC volume $\left(\mathrm{mm}^{3}\right)$, temporalis volume (muscle volume, $\mathrm{mm}^{3}$ ), and the TAC volume to muscle volume ratio (TAC:muscleR, $\%)$.

ICCs were computed to measure intra-rater reliability for muscle and TAC volumes. ICCs less than 0.4 , between 0.4 and 0.75 , and greater than 0.75 were considered poor, fair to good, and excellent, respectively.

A multivariate analysis of covariance (MANCOVA) was used to test the effect of sex, age, and the interaction sex-by-age on muscle and TAC volumes. MANCOVA was used as muscle, and TAC volumes (dependent variables) were positively correlated (Spearman's $\rho=0.852, p<0.001)$. Sex was included in the model as a fixed factor (categorical variable), and age as a covariate (continuous variable). The inclusion of the interaction sex-by-age in the statistical model allowed to account for a potential effect of different age entry in puberty between male and female individuals.

A univariate analysis of covariance (ANCOVA) was used to test theeffect of sex, age, and the interaction sex-by-ageon TAC:muscleR.

The normality of standardized residuals was tested using the Kolgomorov-Smirnov test. Since residuals of muscle and TAC volumes were not normally distributed, these measures were logarithmically transformed.

A minimum sample size of $72 \mathrm{MRI}$ scans was required to obtain $80 \%$ power with a small effect size $\left(f^{2}=0.10, \alpha=0.05\right)$ considering the abovementioned factor (sex) and covariate (age), and their interaction.

All statistical analyses were performed in SPSS Statistics for Windows (Version 24.0., IBM, Armonk, NY) by a single operator (I.C.) who was blinded to age and sex of MRI studies (dataset masking). The level of statistically significance was set at $p<0.05$.

\section{Results}

The intra-rater reliability was excellent for both the temporal muscle and its TAC (muscle: ICC: 0.989, 95\%CI $=0.96-0.99, p<0.001$; TAC: ICC: 0.98 ; $95 \% \mathrm{CI}=0.95-$ $0.99, p<0.001)$. Descriptive statistics for temporal muscle
Isidro/Cioffi 


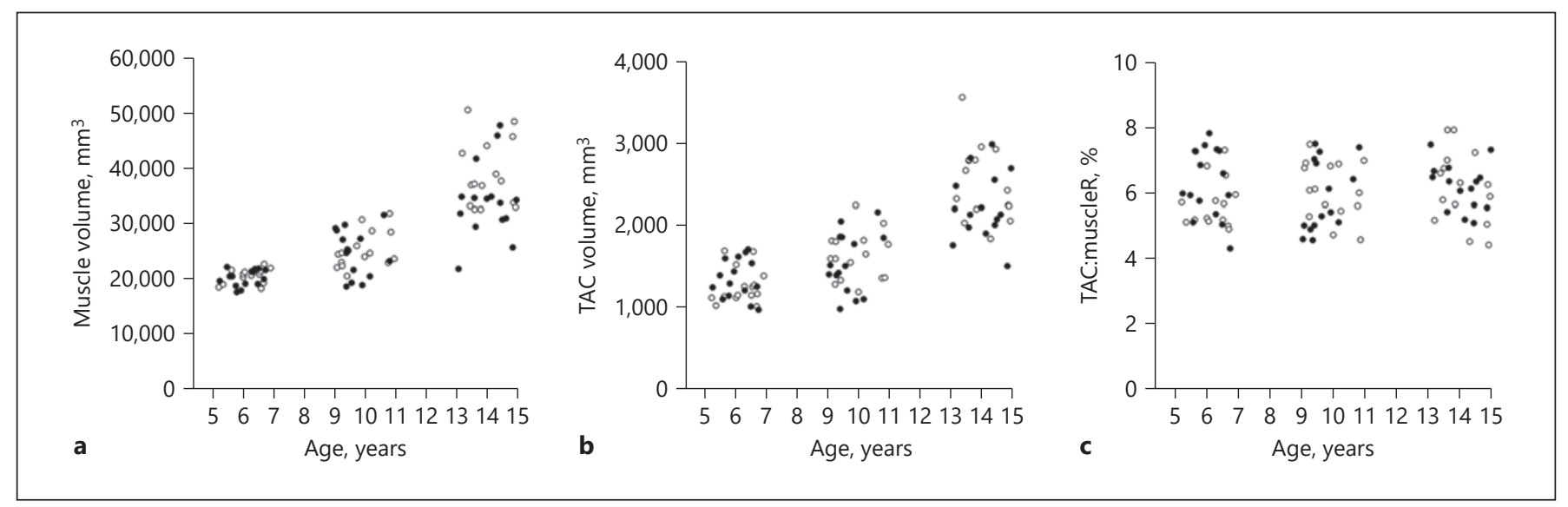

Fig. 2. Scatter plots showing temporalis volumes (a), TAC volumes (b), and TAC:muscleR (c) across ages in 90 MRI scans of male (white) and female (black) subjects.

Table 2. Results from the Multivariate Analysis of Covariance (MANCOVA)

\begin{tabular}{llrrr}
\hline Independent variables & Dependent variables & $f$ value & $p$ value & Partial $\eta^{2}$ \\
\hline \multirow{2}{*}{ Sex } & Temporalis volume & 1.328 & 0.252 & 0.015 \\
& TAC volume & 1.839 & 0.179 & 0.021 \\
\hline Age & Temporalis volume & 240.213 & $<\mathbf{0 . 0 0 1}$ & 0.736 \\
& TAC volume & 134.455 & $<\mathbf{0 . 0 0 1}$ & 0.610 \\
\hline Sex*Age & Temporalis volume & 3.154 & 0.079 & 0.035 \\
& TAC volume & 2.842 & 0.095 & 0.032 \\
\hline
\end{tabular}

* Measures of dependent variables were logarithmically transformed. TAC, tendon-aponeurosis complex. Bold type: statistically significant at $p<0.05$.

and TAC volumes, and TAC:muscleR are reported in Table 1. Distributions of muscle and TAC volumes, as well as TAC:muscleR values for all MRI studies are depicted in Figure 2.

Results from the MANCOVA are reported in Table 2. The volume of the temporalis as well as its TAC increased with age (muscle: $B=0.063, p<0.001-y o u n g$ children: $20,356.44 \pm 1,365.54 \mathrm{~mm}^{3}$; children: $24,934.20 \pm 3,731.56$ $\mathrm{cm}^{3}$; adolescents: $36,418.80 \pm 6,714.89 \mathrm{~mm}^{3}$; TAC: $B=$ $0.063, p<0.001-$ young children: $1,303.21 \pm 229.08 \mathrm{~mm}^{3}$; children: 1,583.10 $\pm 328.12 \mathrm{~mm}^{3}$; adolescents: 2,369.05 \pm $\left.447.15 \mathrm{~mm}^{3}\right)$. Muscle and TAC volumes were not affected by sex (muscle: $p=0.252$; TAC: $p=0.179$ ) or by the interaction sex-by-age (muscle: $p=0.079$; TAC: $p=0.095$ ).

Results from the ANCOVA are reported in Table 3. TAC:muscleR did not change significantly with age $(p=$ 0.655; young children: $6.01 \pm 0.95 \%$; children: $5.98 \pm$
0.97\%; adolescents: $6.17 \pm 0.91 \%)$, and was not affected by sex $(p=0.438)$ or by the interaction sex-by-age $(p=$ $0.524)$.

\section{Discussion and Conclusion}

In this cross-sectional study, we measured the volumes of the temporalis TAC and the TAC:muscleR (\%) in healthy male and female individuals aged between 5 and 15 years old and tested whether there were sex differences. To do so, we collected MRI studies from the CMI$\mathrm{HBN}$ data repository. Based on the age ranges we selected, it is possible to assume that the study included individuals with full primary or early mixed dentition (young children, 5-7 years old), mixed dentition (children, 9-11 years old), and permanent dentition (adolescents, 13-15 
Table 3. Results from the Analysis of Covariance (ANCOVA)

\begin{tabular}{lccl}
\hline Independent variables & $f$ value & $p$ value & Partial $\eta^{2}$ \\
\hline Sex & 0.607 & 0.438 & 0.007 \\
Age & 0.201 & 0.655 & 0.002 \\
Sex*Age & 0.409 & 0.524 & 0.005 \\
\hline
\end{tabular}

* The tendon-aponeurosis complex (TAC) to temporal muscle ratio (TAC:muscleR) was included as dependent variable.

years old). We found that the volumes of the temporalis and its TAC increase with age, that the TAC:muscleR remained stable from 5 to 15 years, and that there were no significant differences between male and female individuals.

The morphological appearance of the temporalis TAC among all participants remained consistent. The TAC was thicker at its attachment on the coronoid process and became thinner cranially. It divided the muscle into 2 clear anatomical compartments, i.e., superficial and deep temporalis, in agreement with other reports [Geers et al., 2005; Lee et al., 2012]. Other anatomical muscle compartments were not evident. Of note, children presented less evident aponeuroses (in all 3 planes of the space) compared to adolescents. In all individuals, the TAC became less distinguishable in cranial direction as the MR signal was weaker, and the superficial and deep temporalis were intermingled in the superior part of the muscle.

Chang et al. [2013] assessed the intramuscular innervations of the temporalis in 3 dimensions using dissection, direct digitization, and reconstruction in 10 cadavers of adult individuals. They found 5 functional compartments, 3 of which were in the superior part of the muscle above the mandibular ramus, and 2 below it. In our MRI studies, we could not identify more than 2 anatomical compartments (superficial and deep temporalis), which likely indicates that the anatomical compartmentalization of the temporalis may not overlap its functional compartmentalization. However, comparisons between studies may be limited since our study assessed children and adolescents.

To the best of our knowledge, this is the first study providing quantitative information about the temporalis TAC in male and female children and adolescents, which makes comparisons with other studies limited. Our data indicate that the volumes of the temporalis and its TAC increase with age, and that the TAC:muscleR does not change significantly over time. The volumetric data of the adolescents of this study are within the range of those retrieved in our previous study [Moayedi et al., 2020], in which the temporalis was measured in 2 independent cohorts of healthy adults recruited in Sydney (12 females, 5 males; mean age: $42.0 \pm 17.0$ years) and Toronto (17 females; mean age \pm SD $32.8 \pm 9.8$ years). In fact, the mean \pm SD temporalis volumes in that study were $33,466.48 \pm$ $6,809.27 \mathrm{~mm}^{3}$ and $31,534.67 \pm 6,592.86 \mathrm{~mm}^{3}$, respectively. The mean \pm SD TAC volumes were $2,092.49 \pm 498.20$ $\mathrm{mm}^{3}$ and $3,910.41 \pm 989.93 \mathrm{~mm}^{3}$, respectively. In the current study, the volumes of the temporalis and its TAC in adolescents were $36,418.80 \pm 6,714.89 \mathrm{~mm}^{3}$ and 2,369.05 $\pm 447.15 \mathrm{~mm}^{3}$. Of interest, in the MRI studies of the Sydney cohort [Moayedi et al., 2020], which had an isotropic voxel size, the TAC-to-temporalis ratio was $6.40 \pm 1.74 \%$, which aligns with the value (about 6\%) found in this study.

Since craniofacial growth studies indicate that growth of the neurocranium is mostly completed by the age of 15 , with minimal further growth occurring after 10 years [Kamdar et al., 2009; Frassanito et al., 2019], and the TAC:muscleR we measured in this study and the previous study in adults [Moayedi et al., 2020] are similar, it is possible that the TAC:muscleR remains stable from childhood to adulthood. This finding is of particular relevance, as individuals with chronic pain due to mTMD present with a reduced temporalis TAC:muscleR [Moayedi et al., 2020], which hinders the ability of the muscle to dissipate forces generated during function [Fiorentino and Blemker, 2014]. It is possible that the reduced TAC:muscleR observed in patients with mTMD may preexist the onset of $\mathrm{mTMD}$, and predispose these patients to muscle injury. Nonetheless, it is equally feasible that mTMD leads to TAC atrophy [Moayedi et al., 2020]. The possibility that the temporalis TAC:muscleR remains stable from childhood to adulthood in healthy individuals is particularly valuable, as it suggests that a reduced TAC:muscleR may be a potential candidate biomarker of chronic mTMD. However, future studies are needed to test this hypothesis.

We did not find a significant effect of sex on all outcome measures. To the best of our knowledge, our study is the first to examine sex differences in temporalis TAC volumes across different ages. Therefore, we cannot make direct comparisons with the available literature. There is some evidence that male individuals present slightly larger head circumference than females during childhood and adolescence [Roche et al., 1997], and that bite force is greater in male than females from 7 to 17 years old [Roldán et al., 2016], both of which may indicate that the temporal 
muscle may be larger in males than females of the same age. Yet, the morphology of the temporalis is also related to mandibular morphology [Sella-Tunis et al., 2018]. Of importance, sexual dimorphism in mandibular morphology is minimal from 4 to 14 years of age [Coquerelle et al., 2011]. Therefore, it is possible that sex differences in temporalis muscle and TAC morphology may become evident after the age of 14 .

Several studies have suggested that the viscoelastic properties of tendon and aponeuroses differ between male and female individuals, which could impact on the ability to adapt to exercise. Kubo et al. [2003] investigated the mechanical properties of the medial gastrocnemius muscle and found that stiffness and Young's modulus were significantly lower in female than male individuals. Using MRI, Magnusson et al. [2007] measured the patellar tendon-cross sectional area (CSA) for trained and untrained men and women. Trained men had significantly larger patellar tendon CSA than untrained men. Yet, a similar difference was not detected between trained and untrained women [Magnusson et al., 2007]. Of interest, resting fractional synthesis rate of collagen was lower in women than men $72 \mathrm{~h}$ after exercise which indicates that females show reduced adaptability of tendon properties to extended exercise [Seynnes et al., 2011]. Therefore, sex-related differences in tendon structural properties could explain why female are more susceptible to overuse musculoskeletal injuries than males [Kuboet al., 2003; Maffulli et al., 2003]. Hence, further studies should focus on understanding whether the adaptation mechanisms of the temporalis TAC to mechanical loading are different between male and female individuals.

This study has some limitations. We used a publicly available MRI dataset and did not recruit research participants directly. Hence, we could not perform a clinical examination and collect information about dental maturation, and craniofacial and mandibular morphology of research participants. As this information was not collected by the investigators who created the CMI-HBN MRI data repository, we could not include it as potential confounder in our analysis. Similarly, as we could not collect data (e.g., preferred chewing side, asymmetric malocclusions, etc.) that could account for possible muscle asymmetry and be used as covariates in the statistical analysis, we chose the right side as a convenient ROI for our segmentations. However, the information available indicated that all participants were healthy and did not have orofacial pain conditions. Further studies could be designed to determine the relationship between craniofacial and mandibular morphology on the temporalis TAC morphometry. Second, given the similar MR signal intensities, it was impossible to discern the temporalis tendon from the cortical bone at the attachment sites on the mandible. To address this limitation, characterization of the inferior boundary of the TAC was by the first axial slice where the MR signal from the cancellous bone of the coronoid process appeared, as done in our previous study in adults [Moayedi et al., 2020]. Third, the limited resolution of the MRI data of the CMI-HBN data repository used in this study may have contributed to partial volume effect artifacts, which could have affected the evaluation of segmented tissues. The use of smaller voxels could have reduced the occurrence of these artifacts. However, this was the first attempt to collect information on the temporalis muscle TAC of young individuals using a publicly available MRI dataset, which was primary collected for brain imaging.

In conclusion, we have provided, for the first time, volumetric data of the temporalis TAC in male and female children and adolescents. Our findings indicate that the temporalis and its TAC increase their size until the age of 15 , while the TAC-to-temporal muscle ratio remains stable. We also demonstrated that that the volume of the temporalis and its TAC are not affected by sex until the age of 15 . Given the potential role of the temporalis TAC in mTMD [Moayedi et al., 2020] and the higher prevalence of TMD in adult females [Slade et al., 2016], further studies should be planned to study sexrelated differences in temporalis morphometric parameters in adults and investigate whether the mechanical properties of the temporalis TAC and its capacity to adapt to mechanical loads differs between male and female individuals.

\section{Acknowledgement}

We are grateful to Dr. Gaurav Krishnamoorthy (Oral Radiologist, Princess Margaret Hospital, Toronto, ON, Canada) for providing assistance.

\section{Statement of Ethics}

The research was conducted ethically in accordance with the World Medical Association Declaration of Helsinki.The research study was reviewed and approved by the University of Toronto Research Ethics Board (\#37065) and the Child Mind InstituteHealthy Brain Network (CMI-HBN). There was no active recruitment of participants as the analysis was based on the publicly available CMI-HBN Magnetic resonance imaging (MRI) database. 


\section{Conflict of Interest Statement}

The authors declare no conflict of interest.

\section{Funding Sources}

This study was supported by the Summer Research Program at the Faculty of Dentistry, University of Toronto, ON, Canada.

\section{Author Contributions}

R.I. developed the research design, collected and analyzed the MRI studies, and drafted the manuscript. I.C. conceptualized the study, developed the research design, performed the statistical analysis, and drafted the manuscript.

\section{References}

Benoliel R, Svensson P, Heir GM, Sirois D, Zakrzewska J, Oke-Nwosu J, et al. Persistent orofacial muscle pain. Oral Dis. 2011 Apr; 17(17 Suppl 1):23-41.

Blanksma NG, van Eijden TM. Electromyographic heterogeneity in the human temporalis and masseter muscles during static biting, open/ close excursions, and chewing. J Dent Res. 1995 Jun;74(6):1318-27.

Blanksma NG, van Eijden TM, van Ruijven LJ, Weijs WA. Electromyographic heterogeneity in the human temporalis and masseter muscles during dynamic tasks guided by visual feedback. J Dent Res. 1997 Jan;76(1):542-51.

Chang Y, Cantelmi D, Wisco JJ, Fattah A, Hannam AG, Agur AM. Evidence for the functional compartmentalization of the temporalis muscle: a 3-dimensional study of innervation. J Oral Maxillofac Surg. 2013 Jul;71(7):1170-7.

Coquerelle M, Bookstein FL, Braga J, Halazonetis DJ, Weber GW, Mitteroecker P. Sexual dimorphism of the human mandible and its association with dental development. Am J Phys Anthropol. 2011 Jun;145(2):192-202.

Farella M, Palla S, Erni S, Michelotti A, Gallo LM. Masticatory muscle activity during deliberately performed oral tasks. Physiol Meas. 2008 Dec;29(12):1397-410.

Fillingim RB, Maixner W. Sex-related factors in temporomandibular disorders. In: Fillingim RB, editor. Sex, Gender, and Pain. Progress in Pain Research and Management. Seattle: IASP Press; 2000. p. 309-25.

Fiorentino NM, Blemker SS. Musculotendon variability influences tissue strains experienced by the biceps femoris long head muscle during high-speed running. J Biomech. 2014 Oct;47(13):3325-33.
Frassanito P, Bianchi F, Pennisi G, Massimi L, Tamburrini G, Caldarelli M. The growth of the neurocranium: literature review and implications in cranial repair. Childs Nerv Syst. 2019 Sept;35(9):1459-65.

Geers C, Nyssen-Behets C, Cosnard G, Lengelé B. The deep belly of the temporalis muscle: an anatomical, histological and MRI study. Surg Radiol Anat. 2005 August;27(3):184-91.

Huang GJ, LeResche L, Critchlow CW, Martin MD, Drangsholt MT. Risk factors for diagnostic subgroups of painful temporomandibular disorders (TMD). J Dent Res. 2002 Apr; 81(4):284-8.

Kamdar MR, Gomez RA, Ascherman JA. Intracranial volumes in a large series of healthy children. Plast Reconstr Surg. 2009 Dec; 124(6):2072-5.

Kubo K, Kanehisa H, Fukunaga T. Gender differences in the viscoelastic properties of tendon structures. Eur J Appl Physiol. 2003 Feb; 88(6):520-6.

Lee JY, Kim JN, Kim SH, Choi HG, Hu KS, Kim $\mathrm{HJ}$, et al. Anatomical verification and designation of the superficial layer of the temporalis muscle. Clin Anat. 2012 Mar;25(2):176-81.

Maffulli N, Wong J, Almekinders LC. Types and epidemiology of tendinopathy. Clin Sports Med. 2003 Oct;22(4):675-92.

Magnusson SP, Hansen M, Langberg H, Miller B, Haraldsson B, Westh EK, et al. The adaptability of tendon to loading differs in men and women. Int J Exp Pathol. 2007 Aug;88(4): 237-40.

Michelotti A, Cioffi I, Festa P, Scala G, Farella M. Oral parafunctions as risk factors for diagnostic TMD subgroups. J Oral Rehabil. 2010 Mar; 37(3):157-62.
Moayedi M, Krishnamoorthy G, He PT, Agur A Weissman-Fogel I, Tenenbaum HC, et al. Structural abnormalities in the temporalis musculo-aponeurotic complex in chronic muscular temporomandibular disorders. Pain. 2020 Aug;161(8):1787-97.

Roche JJ, Cisneros GJ, Acs G. The effect of acetaminophen on tooth movement in rabbits. Angle Orthod. 1997;67(3):231-6.

Roldán SI, Restrepo LG, Isaza JF, Vélez LG, Buschang PH. Are maximum bite forces of subjects 7 to 17 years of age related to malocclusion?. Angle Orthod. 2016 May;86(3):456-61.

Sella-Tunis T, Pokhojaev A, Sarig R, O'Higgins P, May H. Human mandibular shape is associated with masticatory muscle force. Sci Rep. 2018 April;8(1):6042.

Seynnes OR, Koesters A, Gimpl M, Reifberger A, Niederseer D, Niebauer J, et al. Effect of alpine skiing training on tendon mechanical properties in older men and women. Scand J Med Sci Sports. 2011 Aug;21(Suppl 1):39-46.

Sharma P, Maffulli N. Biology of tendon injury: healing, modeling and remodeling. J Musculoskelet Neuronal Interact. 2006 Apr-Jun; 6(2):181-90.

Slade GD, Ohrbach R, Greenspan JD, Fillingim RB, Bair E, Sanders AE, et al. Painful Temporomandibular Disorder: Decade of Discovery from OPPERA Studies. J Dent Res. 2016 Sep;95(10):1084-92.

Yushkevich PA, Piven J, Hazlett HC, Smith RG, Ho S, Gee JC, et al. User-guided 3D active contour segmentation of anatomical structures: significantly improved efficiency and reliability. Neuroimage. 2006 Jul;31(3):111628. 